\title{
A MULTILINEAR VERSION OF THE MARCINKIEWICZ INTERPOLATION THEOREM
}

\author{
ROBERT S. STRICHARTZ ${ }^{1}$
}

It is natural to ask if there exists a multilinear version of the Marcinkiewicz interpolation theorem [3] corresponding to the multilinear Riesz interpolation theorem. If a bilinear transformation $T(f, g)$ is defined from

$$
\left\{L^{p_{0}}(X, \mu) \times L^{q_{0}}(Y, \nu)\right\}+\left\{L^{p_{1}}(X, \mu) \times L^{q_{1}}(Y, \nu)\right\}
$$

to measurable functions on $(Z, \sigma)$ and satisfies the weak-type estimates $\left(p_{i}, q_{i} ; r_{i}\right)$ :

$$
\sigma\{z:|T(f, g)(z)| \geqq \alpha\} \leqq M_{i}\left(\frac{\|f\|\left\|_{p_{i}}\right\| g \|_{q_{i}}}{\alpha}\right) r_{i}
$$

for $i=0,1$, does it follow that it satisfies the strong-type estimate $(p, q ; r):\|T(f, g)\|_{r} \leqq K M_{1}^{1-t} M_{2}^{t}\|f\|_{p}\|g\|_{q}$ where

$$
\frac{1}{p}=\frac{1-t}{p_{0}}+\frac{t}{p_{1}}, \quad \frac{1}{q}=\frac{1-t}{q_{0}}+\frac{t}{q_{1}}, \quad \frac{1}{r}=\frac{1-t}{r_{0}}+\frac{t}{r_{1}}
$$

if $p \leqq r, q \leqq r$ and $0<t<1$ ? The answer is no, as shown by the following counterexample:

Let $X=Y=Z=(0, \infty)$ and $\mu=\nu=\sigma=$ Lebesgue measure. Let $T(f, g)(z)=\int_{0}^{\infty} f(x z) g(x) d x$. Then

$$
|T(f, g)(z)| \leqq\|f(x z)\|\left\|_{p}\right\| g(x)\left\|_{q}=z^{-1 / p}\right\| f\left\|_{p}\right\| g \|_{q}
$$

where $1 / p+1 / q=1$ hence we have the weak-type $(p, q ; p)$ estimate holding for all $p, 1 \leqq p \leqq \infty$. But we never have the strong-type estimate (unless $p=\infty$ ). For if we choose $g$ to be a positive function and consider the linear operator

$$
S(f)(z)=\int_{0}^{\infty} f(x z) g(x) d x=\int_{0}^{\infty} f(x) g\left(\frac{x}{z}\right) \frac{d x}{z},
$$

it is an integral operator with positive kernel homogeneous of degree -1 , hence by Hardy-Littlewood-Pólya [2, Chapter 9] will be bounded in $L^{p}$ if and only if $\int_{0}^{\infty} g(x) x^{-1 / p} d x<\infty$ which is not true for every $g \in L^{q}$.

Received by the editors July 20, 1967 and, in revised form, February 19, 1968.

1 This work was done while the author held a NATO Postdoctoral Fellowship at the Faculté des Sciences. Orsay, France. 
If we are willing to strengthen the hypotheses, however, by adding a third point, we can obtain the desired conclusion as follows:

THEOREM. Let $T(f, \cdots, g)$ be an m-linear transformation from $\left\{L^{p_{0}}(X, \mu) \times \cdots \times L^{q_{0}}(Y, \nu)\right\}+\cdots+\left\{L^{p_{m}}(X, \mu) \times \cdots \times L^{q_{m}}(Y, \nu)\right\}$ to measurable function on $(Z, \sigma)$. Suppose $T$ satisfies the $m+1$ weaktype $\left(p_{i}, \cdots, q_{i} ; r_{i}\right)$ estimates for $i=0, \cdots, m$. Suppose the $m+1$ points $\left(1 / p_{i}, \cdots, 1 / q_{i}\right)$ in $m$-space span a nondegenerate simplex, and let $(1 / p, \cdots, 1 / q)$ be a point in the interior of the simplex. In barycentric coordinates

$$
\frac{1}{p}=\sum \frac{s_{i}}{p_{i}}, \cdots, \frac{1}{q}=\sum \frac{s_{i}}{q_{i}}
$$

where $\sum s_{i}=1$ and $0<s_{i}<1$. Let $1 / r=\sum s_{i} / r_{i}$. Suppose that for just one of the $p, \cdots, q$ variables, say $p$, we have $p \leqq r$ and that $r$ is nonconstant as $p$ varies with the other variables fixed. Then $T$ satisfies the strong-type $(p, \cdots, q ; r)$ estimate.

For simplicity we will consider only the case of bilinear transformations. The proof in the general case is entirely analogous. We need the following lemma, which follows from the work of Calderón [1, Theorems 10.1 and 13.6] in case $r_{0}, r_{1}>1$. We shall give a proof suggested by E. M. Stein. In what follows we use the properties of Banach space valued analytic functions [4] and Lorentz spaces [5].

LEMMA. Let $T$ be a bilinear transformation defined on simple functions satisfying a weak-type $\left(p_{i}, q_{i} ; r_{i}\right)$ estimate with constant $M_{i}$ for $i=0,1$, where $p_{i}, q_{i}, r_{i} \geqq 1$ and $r_{0} \neq r_{1}$. Then $T$ satisfies a weak-type $(p, q ; r)$ estimate with constant $M_{0}^{1-\imath} M_{1}^{\imath}$.

Proof. If $f$ is any measurable function on $(Z, \sigma)$ such that

$$
\sigma\{z:|f(z)|>\alpha\}
$$

is finite for all $\alpha>0$, let $f^{*}(t)=\sup \{\alpha: \sigma\{z:|f(z)|>\alpha\} \geqq t\}$ be the nonincreasing rearrangement of $f$. Let $\|f\|_{r, \infty}^{*}=\sup _{t>0} t^{1 / r} f^{*}(t)$ and $\|f\|_{r, 1}^{*}=\int_{0}^{\infty} t^{1 / r-1} f^{*}(t) d t$, and denote by $L^{r, \infty}$ and $L^{r, 1}$ the spaces of functions with $\|f\|_{r, \infty}^{*}$ and $\|f\|_{r, 1}^{*}$ finite. Although these are not norms, they are equivalent to norms if $r>1$, and $L^{r, \infty}$ is the dual space of $L^{r^{\prime}, 1}$, where $1 / r+1 / r^{\prime}=1$ (note $L^{\infty, \infty}=L^{\infty}$ and $L^{1,1}=L^{1}$ ). Furthermore

$$
\|f\|_{r, \infty}^{*}=\sup \left\{\left|\int f g d \sigma\right|:\|g\|_{r^{\prime}, 1}^{*} \leqq 1\right\}[5] \text {. }
$$


It is easy to see that a weak-type $(p, q ; r)$ estimate is equivalent to $\|T(f, g)\|_{r, \infty}^{*} \leqq M\|f\|_{p}\|g\|_{q}$. We note that

$$
\|T(f, g)\|_{r, \infty}^{*}=\left(\left\||T(f, g)|^{1 / 2}\right\|_{2 r, \infty}^{*}\right)^{2} \quad \text { and } 2 r>1 \text {. }
$$

Thus to prove the lemma we must show

$$
\int|T(f, g)|^{1 / 2} h d \sigma \leqq\left(M_{0}^{1-t} M_{1}^{t}\right)^{1 / 2}
$$

for $\|f\|_{p} \leqq 1,\|g\|_{q} \leqq 1$ and $\|h\|_{u, 1} \leqq 1$ where $1 / u+1 / 2 r=1$, given that the same inequality holds for $t=0$ and $t=1$.

The proof now proceeds as the proof of the Riesz-Thorin theorem, except that some modifications are necessary since $|T(f, g)|^{1 / 2}$ is not linear. We may assume $(Z, \sigma)$ is nonatomic, since otherwise we may replace each atom $Z_{0}$ by an interval of the same measure and set $T(f, g)=T(f, g)\left(Z_{0}\right)$ on that interval. We let

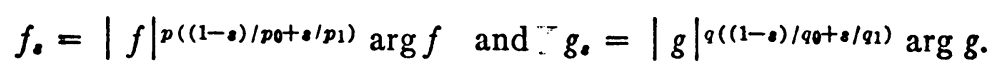

We choose a function $\phi$ on $(Z, \sigma)$ equimeasurable with $1 / t$ on $((0, \sigma(Z)), d t)$ such that $\left(\phi^{\alpha} h\right)^{*}=\left(1 / t^{\alpha}\right) h^{*}(t)$ for every $\alpha>0$. Then let

$$
H_{*}=\phi^{(1-s) / u_{0}+s^{\prime} / u_{1}-1 / u} \cdot h
$$

Finally let

$$
F(s)=\left|M_{0}^{1-s} M_{1}^{s}\right|^{-1 / 2} \int\left|T\left(f_{s}, g_{s}\right)\right|^{1 / 2}\left|H_{s}\right| d \sigma .
$$

It is easy to show that our hypotheses on $T$ imply $|F(i \tau)| \leqq 1$ and $|F(1+i \tau)| \leqq 1$ for all real $\tau$, and the desired conclusion is $|F(t)| \leqq 1$. This will follow from the Phragmén-Lindelöf principle if we can show that $F$ is bounded and subharmonic in the strip $0 \leqq \operatorname{Re}(S) \leqq 1$.

Boundedness is immediate, since $\left|T\left(f_{s}, g_{s}\right)\right|^{1 / 2}$ is bounded in $L^{2 r_{0}, \infty} \cap L^{2 r_{1}, \infty}$ and $H_{s}$ is bounded in $L^{u_{0}, 1}+L^{u_{1}, 1}$. Next we claim that for almost every $z \in Z$, the function $T\left(f_{s}, g_{s}\right)(z)$ is analytic in $s$ (after redefining these functions on sets of measure zero). Indeed $T\left(f_{*}, g_{s}\right)$ is a holomorphic Banach-space valued function (since either $r_{0}$ or $r_{1}$ is greater than 1) hence Cauchy's integral formula

$$
T\left(f_{s}, g_{s}\right)=\frac{1}{2 \pi i} \int_{c} \frac{T\left(f_{\iota}, g_{t}\right)(z)}{s-t} d t
$$

is valid. But 


$$
\frac{1}{2 \pi i} \int_{C} \frac{T\left(f_{t}, g_{t}\right)(z)}{s-t} d t
$$

is holomorphic in $s$ for fixed $z$ provided $\int_{C}\left|T\left(f_{t}, g_{t}\right)(z)\right| d t<\infty$, which holds almost everywhere since $\int_{C} \int_{B}\left|T\left(f_{t}, g_{t}\right)(z)\right| d z d t<\infty$ for every $B C Z$ of finite measure.

Thus $F(s)$ is an integral of square roots of moduli of holomorphic functions, hence is subharmonic.

Proof of Theorem. We apply the lemma to conclude that $T$ satisfies a weak-type $(p, q ; r)$ estimate for every point in the interior of the triangle. We then consider two points $\left(p+\epsilon, q ; r_{1}\right)$ and $\left(p-\epsilon, q ; r_{2}\right)$ in the triangle and apply the ordinary Marcinkiewicz interpolation theorem to $S(f)=T(f, g)$ to obtain the strong $(p, q ; r)$ estimate, noting that we have $p \leqq r$ and $r_{1} \neq r_{2}$ by hypothesis.

\section{REFERENCES}

1. A.-P. Calderón, Intermediate spaces and interpolation, Studia Math. 24 (1964), 113-190.

2. G. H. Hardy, J. E. Littlewood and G. Pólya, Inequalities, 2nd ed., Cambridge Univ. Press, New York, 1952; Russian transl., IL, Moscow, 1948.

3. A. Zygmund, Trigonometric series. II, Cambridge Univ. Press, New York, 1959; Russian transl., "Mir”, Moscow, 1965.

4. E. Hille and R. S. Phillips, Functional analysis and semi-groups, rev. ed., Amer. Math. Soc. Colloq. Publ., Vol. 31, Amer. Math.Soc., Providence, R. I., 1957; Russian transl., IL, Moscow, 1962.

5. R. A. Hunt, On $L(p, q)$ spaces, Enseignement Math. 12 (1967), 249-276.

Massachusetts Institute of Technology 